\title{
Feature-Dependent Sensitive Periods in the Development of Complex Sound Representation
}

\author{
Michele N. Insanally, Hania Köver, Heesoo Kim, and Shaowen Bao \\ Helen Wills Neuroscience Institute, University of California, Berkeley, California 94720
}

\begin{abstract}
Simple tonal stimuli can shape spectral tuning of cortical neurons during an early epoch of brain development. The effects of complex sound experience on cortical development remain to be determined. We exposed rat pups to a frequency-modulated (FM) sweep in different time windows during early development, and examined the effects of such sensory experience on sound representations in the primary auditory cortex (AI). We found that early exposure to a FM sound resulted in altered characteristic frequency representations and broadened spectral tuning in AI neurons, whereas later exposure to the same sound only led to greater selectivity for the sweep rate and direction of the experienced FM sound. These results indicate that cortical representations of different acoustic features are shaped by complex sounds in a series of distinct sensitive periods.
\end{abstract}

\section{Introduction}

Cortical sound representation is remarkably adaptive early in life, during a "critical period" (Zhang et al., 2002; de Villers-Sidani et al., 2007). Previous examinations of the "critical period" in the auditory cortex have typically used simple tonal stimuli. Repeated exposure of rat pups to a tone, for instance, has been shown to selectively enlarge cortical representation of the tone and alter perceptual behaviors (Zhang et al., 2001; Chang and Merzenich, 2003; de Villers-Sidani et al., 2007; Han et al., 2007). However, probing cortical plasticity with a single-frequency tone might not reveal the full complexity and dynamics of critical period plasticity. Natural, biologically important sounds are generally complex in spectrotemporal structures (Singh and Theunissen, 2003). Psychophysical studies indicate that early experience of complex sounds has a profound impact on auditory perception and perceptual behaviors. Experience with speech, for instance, shapes language-specific phonemic perception, enhancing perceptual contrasts of native speech sounds and reducing perceptual contrasts of some foreign speech sounds (Werker and Tees, 1984; Kuhl et al., 1992; Kuhl et al., 2006). At the electrophysiological level, auditory cortical neurons preferentially respond to certain complex sounds, such as species-specific animal vocalizations (Wang and Kadia, 2001). It is unclear how such selectivity for complex sounds emerges, and whether it is innate or shaped by early experience.

Complex sounds are processed in the auditory cortex by neurons that are selective for sound features on multiple acoustic dimensions, such as spectral composition, amplitude modulation, and frequency modulation (Nelken and Versnel, 2000; Lof-

Received Nov. 4, 2008; revised Feb. 26, 2009; accepted March 30, 2009.

The work was supported by National Institutes of Health Grants DC007883 and DC009259. M.N.I. and H. Kim are supported by National Science Foundation Graduate Research Fellowship Program. H. Köver is supported by Boehringer Ingelheim Fonds Fellowship. We thank Edward Chang and Amy Finn for comments on the manuscript.

Correspondence should be addressed to Dr. Shaowen Bao, Helen Wills Neuroscience Institute, University of California, Berkeley, CA 94720-3190. E-mail: sbao@berkeley.edu.

D0I:10.1523/JNEUROSCI.5311-08.2009

Copyright $\odot 2009$ Society for Neuroscience $\quad$ 0270-6474/09/295456-07\$15.00/0 tus and Sutter, 2001; Lu et al., 2001; Linden et al., 2003; Atencio et al., 2007). While previous studies have shown that spectral and temporal selectivity are shaped by sensory inputs during the critical period (Zhang et al., 2001; Chang and Merzenich, 2003; Chang et al., 2005; de Villers-Sidani et al., 2007; Han et al., 2007), it remains to be determined whether selectivity for complex sound features, such as frequency modulated sweeps, can be altered by early sound exposure.

Earlier studies indicate that selectivity for distinct sound features is performed by different cortical circuits. The characteristic frequency (CF) and the tuning bandwidth (BW), for example, may be determined by thalamocortical and intracortical excitatory connections (Kaur et al., 2004; Metherate et al., 2005; Liu et al., 2007), whereas selectivity for frequency-modulated (FM) sweeps is shaped by intracortical inhibitory circuits underlying the inhibitory receptive field of the neuron (Zhang et al., 2003; Razak and Fuzessery, 2007). These circuits develop in different developmental stages (Gao et al., 1999; Metherate and Aramakis, 1999; Zhang et al., 2001; Arimatsu and Ishida, 2002; Luczyñska et al., 2003; Chang et al., 2005; Larsen and Callaway, 2006), raising the question of whether selectivity for features on different acoustic dimensions is shaped by sensory experience simultaneously or sequentially, and interactively or independently.

To address these questions, we exposed rat pups to downward FM sweeps in different time windows during development, and examined their cortical response properties. Our results indicate that exposure to FM sweeps induces multiple forms of plasticity in sound representations in primary auditory neurons, depending on the time windows of sound exposure. These results indicate that there are multiple feature-dependent sensitive periods in the development of complex sound representation.

\section{Materials and Methods}

Acoustic rearing. All procedures used in this study have been approved by the UC Berkeley Animal Care and Use Committee. Litters of rat pups (female, Sprague Dawley) and their mothers were placed in an anechoic sound-attenuation chamber for a brief period, comprising one or a com- 
A

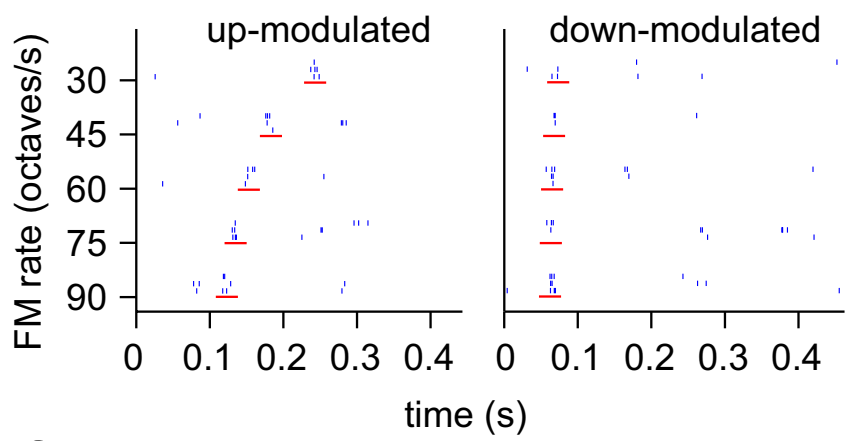

C

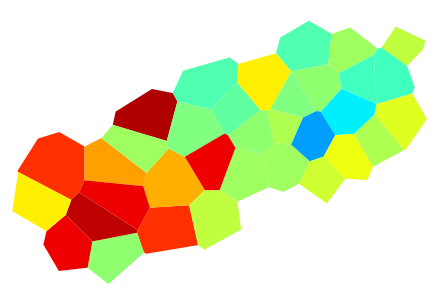

Window 3

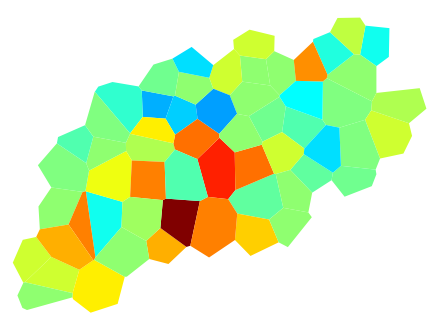

Window 1

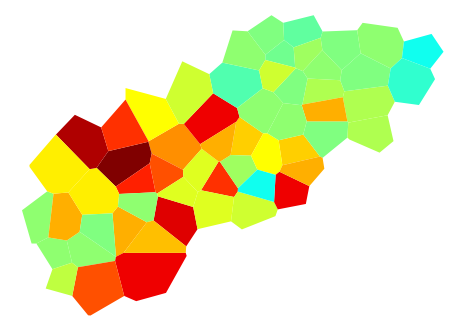

Window 4

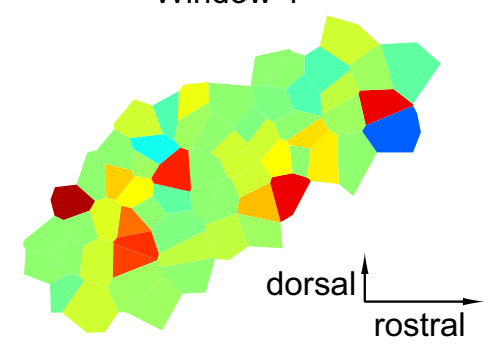

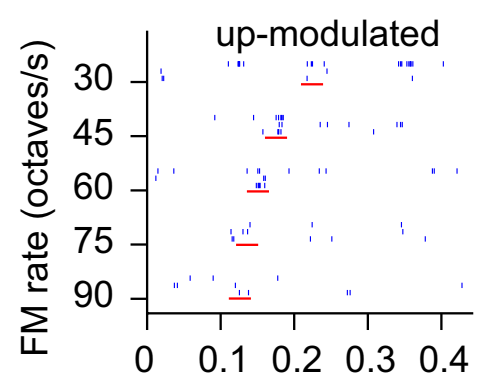

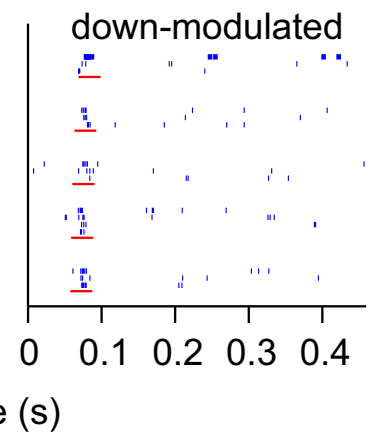

Window 2
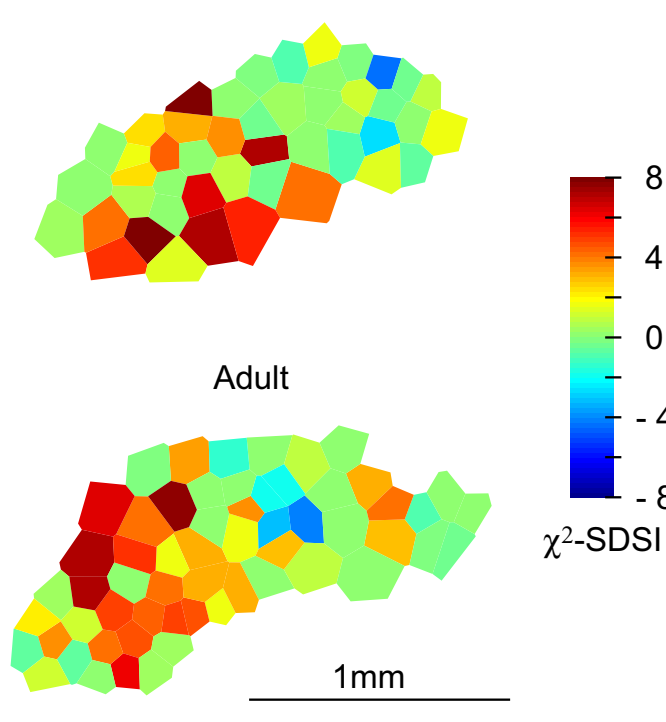

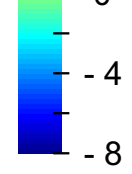

$\chi^{2}-$ SDSI

Figure 1. Exposure to frequency-modulated sounds in the third and fourth time windows alters sweep direction selectivity. $\boldsymbol{A}, \boldsymbol{B}$, Raster plots of responses to FM sounds recorded from high frequency selective neurons in naive and FM-exposed animals, respectively. The red horizontal bars denote the 30 ms response windows. Note the selective response to downward-modulated sounds in $\boldsymbol{B}$. C C Representative cortical maps of the $\chi^{2}$-based sweep direction selectivity. Direction selectivity index was calculated from responses to upward and downward sweeps at a rate of 90 octaves/s. Experimental animals were exposed to downward sweeps in four different time windows. Animals that had heard the downward sweeps in window 4 showed a negative shift of the selectivity index, indicating that the neurons were more selective for downward sweeps than those of the other groups.

bination of four time windows (window 1, P8-P15; window 2, P16-P23; window 3, P24-P31; and window 4, P32-P39). Trains of logarithmic frequency modulated (FM) sweeps (frequency varied exponentially with time from 50 to $0.5 \mathrm{kHz}$ at a rate of -80 octaves $/ \mathrm{s}, 60 \mathrm{~dB}$ SPL, five sweeps in a train at $5 \mathrm{~Hz}$, with $500 \mathrm{~ms}$ quiet periods between trains; all speakers had $<3 \%$ spectral distortion, and their output levels were calibrated for the entire output frequency range) were played to the animals $24 \mathrm{~h} / \mathrm{d}$. In addition, the acoustic environment could also contain vocalization sounds produced by the animals, mainly in a frequency range $>30 \mathrm{kHz}$. After the exposure period, the animals were returned to standard housing conditions.

Electrophysiological recording. The primary auditory cortex of sweepexposed and naive animals were mapped under pentobarbital anesthesia (50 mg/kg for induction, $10-20 \mathrm{mg} / \mathrm{kg}$ supplemental, as needed; intraperitoneal injections) at ages P40 to P60. Animals in different groups were approximately age matched. The primary auditory cortex was defined by its tonotopic organization and reliable neuronal responses to tone pips of selective frequencies. Neurons were evenly sampled from the primary auditory cortex using parylene-coated tungsten microelectrodes (2 M $\Omega$ ) advanced 500-600 $\mu \mathrm{m}$ below the pial surface (layer 4/5), and responses to $25 \mathrm{~ms}$ tone pips of 51 frequencies $(1-32 \mathrm{kHz}, 0.1$ octave spacing) and 8 sound pressure levels $(0-70 \mathrm{~dB}$ SPL, $10 \mathrm{~dB}$ steps $)$ were recorded three times to reconstruct the response-frequency tuning curve. Responses to a series of FM sweeps (frequency range, $0.5-50 \mathrm{kHz}$; sweep rates, 30, 45, 60, 75, and 90 octaves/s, in both up and down directions) were also recorded three times to determine the neurons' selectivity for FM sweep rates. Tone pips and FM sweeps were intermixed and presented in a pseudorandom order.

Analysis. The CF and tuning bandwidth of each neuron were determined automatically, which involves smoothing and thresholding of the receptive field. The CF was calculated as the center of mass of the receptive field. To generate CF maps, Voronoi tessellation was performed to create tessellated polygons, with each polygon corresponding to an electrode penetration site, and assigned the $\mathrm{CF}$ of the site. The magnitudes of responses to FM sweeps were determined in $30 \mathrm{~ms}$ response windows that were automatically set to best fit the response latencies at all the different sweep rates. Under the assumption that the neuronal response was triggered by a specific frequency (denoted as $f$ in $\mathrm{kHz})$ in the FM stimuli, for upsweeps, FM_Rate $\times(T-$ $\left.T_{\min }\right)=\log _{2}\left(f \times 0.5^{-1}\right)$, in which $T$ is the measured latency of the response to the sweep at the FM_Rate, and $T_{\min }$ is the minimal response delay. A regression analysis was performed on FM_Rates and $T$ s to derive $T_{\min }$ and $f$. The expected latency was then calculated as $T_{\exp }=\log _{2}\left(f \times 0.5^{-1}\right) \times$ FM_Rate ${ }^{-1}+T_{\min }$. A $30 \mathrm{~ms}$ window centered at $T_{\exp }$ was used as the response window. The number of spikes in the response window was counted as the response magnitude. Spontaneous firing was determined from the $30 \mathrm{~ms}$ prestimulus period, and was subtracted from the response to 
the FM sweeps. For downsweeps, FM_Rate $X$ $\left(T-T_{\min }\right)=\log _{2}\left(50 \times f^{-1}\right)$, and $T_{\exp }=$ $\log _{2}\left(50 \times f^{-1}\right) \times$ FM_Rate ${ }^{-1}+T_{\text {min }}$. Our visual inspection indicated that the response windows consistently captured the phasic cortical responses to the sweeps (see Fig. 1).

A sweep direction selectivity index (SDSI) was calculated as $\left(R_{\text {up }}-R_{\text {down }}\right) /\left(R_{\text {up }}+R_{\text {down }}\right)$, in which $R_{\mathrm{up}}$ is response magnitude to the upsweep, and $R_{\text {down }}$ is response magnitude to the downsweep. To avoid bias by small responses, we calculated a $\chi^{2}$-based statistic of upsweep selectivity: $\chi^{2}$-SDSI $=\left(R_{\mathrm{up}}-R_{\text {down }}\right) \mid R_{\mathrm{up}}-$ $R_{\text {down }} /\left(R_{\text {up }}+R_{\text {down }}\right)$.

Determining the locations of recorded sites on the tonotopic axis. A line connecting the site with the highest CF and that with the lowest CF was defined as the tonotopic axis. The maps were rotated to orient the tonotopic axis horizontally. For all recorded sites in each map, a linear regression was performed in the form of $\log (\mathrm{CF})=k(X-c)$, in which $\mathrm{CF}$ is the characteristic frequency in $\mathrm{kHz}$, and $X$ is the $x$-coordinate, of the recorded site. Then, $X-c$ is used as the location of the recorded site on the tonotopic axis.

Unless stated otherwise, statistical significance was quantified with ANOVA and post hoc Bonferroni's test using StatView (SAS Institute). Data are presented as mean \pm SEM.

\section{Results}

\section{A sensitive period for cortical FM}

\section{direction selectivity}

Auditory cortical neurons are selective for FM directions at 80 octaves/s, which corresponds to the highest sweep rate at which rats can discriminate sweep directions (Zhang et al., 2003; Gaese et al., 2006). To probe plasticity, we exposed rat pups to downward logarithmic FM sweeps ( 50 to $0.5 \mathrm{kHz}, 60 \mathrm{~dB}$ SPL, -80 octaves per second, 5 sweeps in a train at $5 \mathrm{~Hz}$, with $500 \mathrm{~ms}$ of silence between trains) in one of four time windows (window 1 through window 4, see Materials and Methods). Cortical spectral and FM representations were examined in both sweep-exposed and control animals at ages P40 to P60. Auditory cortical neurons typically responded to the testing FM sweeps with a brief burst of spikes (Fig. $1 A, B$ ). This FM response was quantified using the number of spikes in a $30 \mathrm{~ms}$ response window (automatically determined with an analysis program; for details, see Materials and Methods). To investigate whether direction selectivity was altered by sweep exposure, a sweep direction selectivity index (SDSI) was calculated as $\left(R_{\text {up }}-R_{\text {down }}\right) /$ $\left(R_{\text {up }}+R_{\text {down }}\right)$. Because SDSI can be biased by weak responses, we calculated a $\chi^{2}$ based statistic that measures the significance of sweep direction selectivity: $\chi^{2}$-SDSI $=\left(R_{\mathrm{up}}-\right.$ $\left.R_{\text {down }}\right) \mid R_{\text {up }}-R_{\text {down }} /\left(R_{\text {up }}+R_{\text {down }}\right)$. Neurons selective for upward FM sweeps will have a positive SDSI and a positive $\chi^{2}$-SDSI.
A

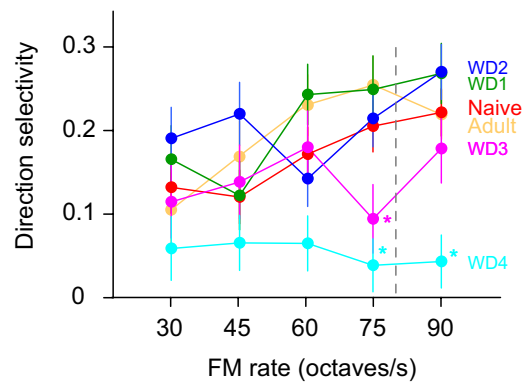

C

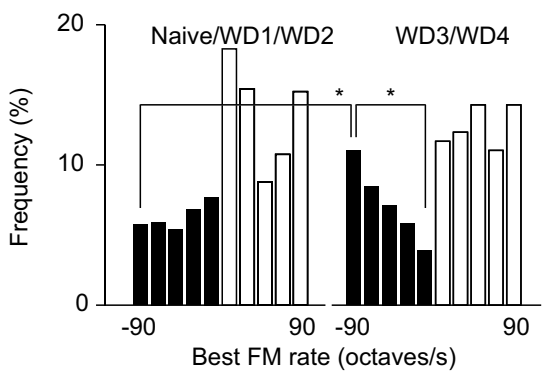

B

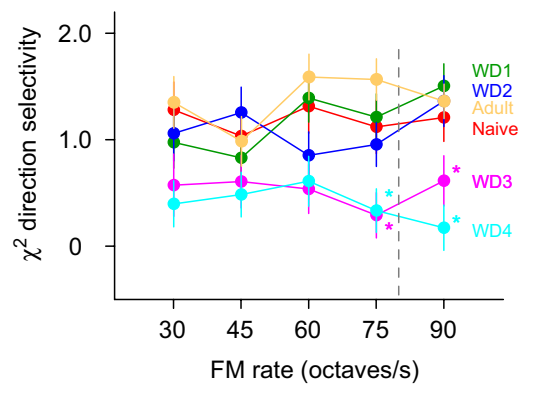

D

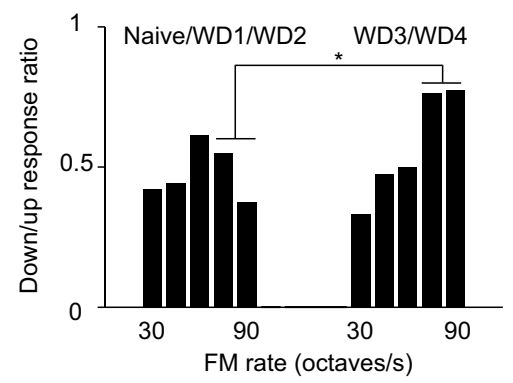

Figure 2. Exposure to frequency-modulated sounds alters sweep direction and rate selectivity. $\boldsymbol{A}$, Sweep direction selectivity index (SDSI) at different sweep rates. Exposure to 80 octaves/s downward FM sounds (marked by the dashed line) in W3 and W4 resulted in downward shifts of SDSI near the exposed sweep rate. $\boldsymbol{B}, \chi^{2}$-based sweep direction selectivity. $\boldsymbol{C}$, Histogram of best FM rates that activate the strongest responses in cortical neurons. More neurons in W3 and W4 animals became tuned to -90 octaves $/ \mathrm{s}$, which is near the exposure FM rate. $\boldsymbol{D}$, The ratio of neuronal responses (number of spikes in a $30 \mathrm{~ms}$ window) to downward versus upward FM sweeps. The ratio is greater for W3 and W4 animals than for the other groups. ${ }^{*} p<0.05$.
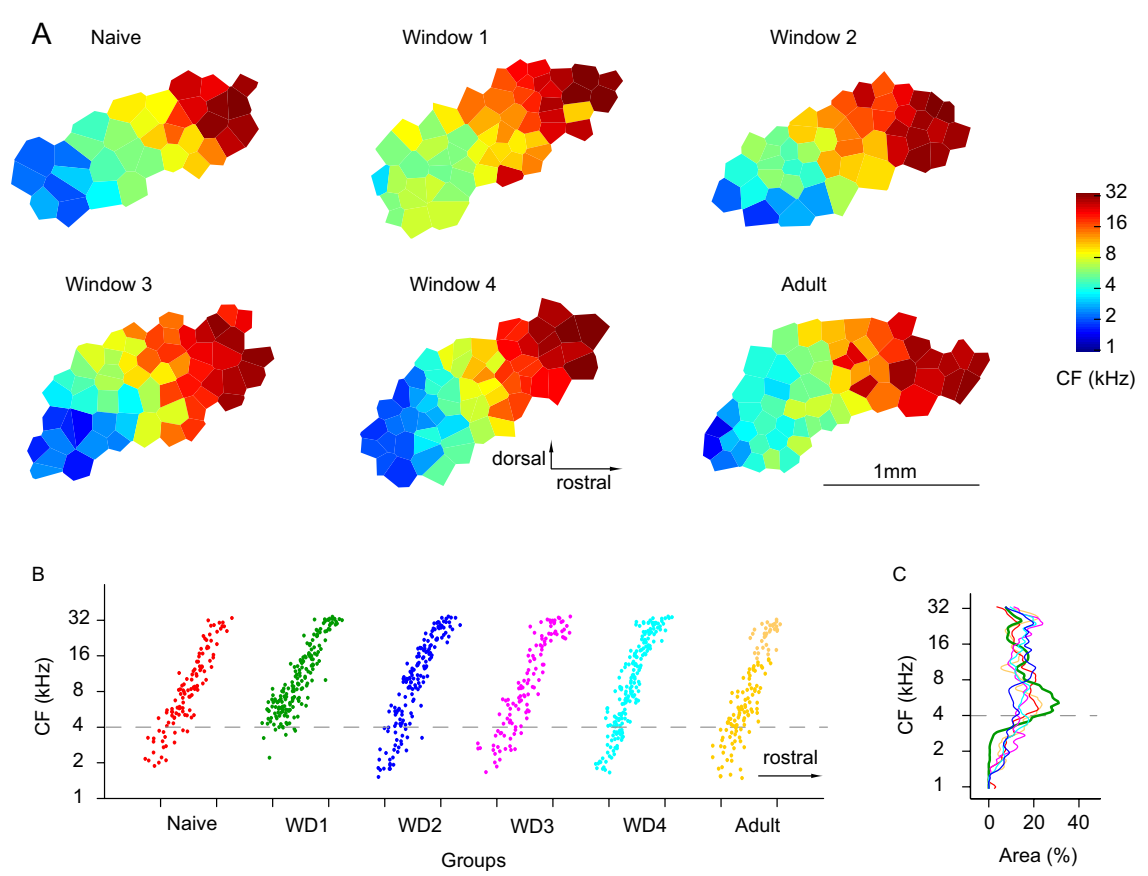

Figure 3. Exposure to downward FM sounds in the first time window alters the cortical CF map. $\boldsymbol{A}$, Representative cortical CF maps of naive, window 1 , window 2 , window 3 , window 4 , and adult animals. $B$, CF distribution along the tonotopic axis. Recordings from four animals were included for each group. $C$, Percentage primary auditory cortical area representing frequencies in a 0.4 octaves frequency band. Color codes for experimental groups are the same as in $\boldsymbol{B}$. Note that only W1 animals had reduced representations of frequencies $<4 \mathrm{kHz}$.
Animals that were exposed to the downward FM sweeps in windows 3 and window 4 showed significantly lower SDSIs and $\chi^{2}$ SDSIs near the exposure FM rate of 80 octaves/s (at 75 and 90 octaves/s) (Fig. $2 A, B)(p<0.05$, ANOVA, compared with all 
A

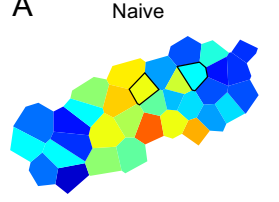

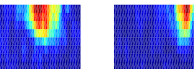
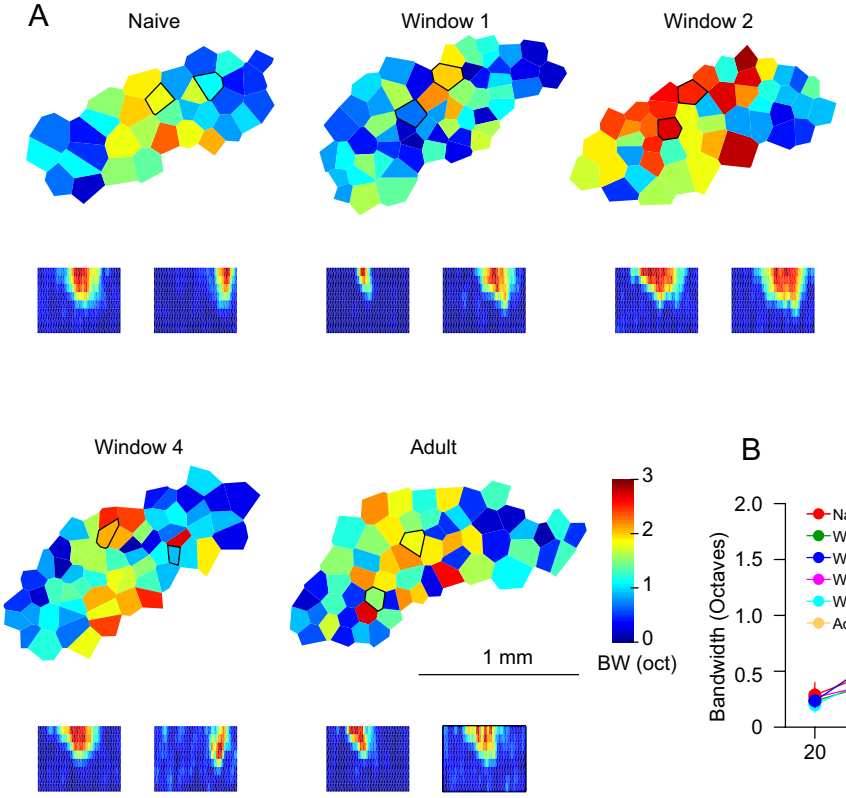

Figure 4. Exposure to downward FM sounds in window 2 broadens frequency tuning. $\boldsymbol{A}$, Representative cortical tuning bandwidth maps based on BWs measured at $70 \mathrm{~dB}$ SPL. Insets are representative receptive fields that had been recorded in the locations outlined in the bandwidth maps. The vertical axis of the receptive field plots depicts sound intensity from 0 to $70 \mathrm{dBSPL}$. The horizontal axis depicts frequencies from 1 to $32 \mathrm{kHz}$. $\boldsymbol{B}$, Tuning bandwidth as a function of sound pressure level. Error bars depict SEM, the majority of which are occluded by the data symbols. Only window 2 animals showed broadened frequency tuning.

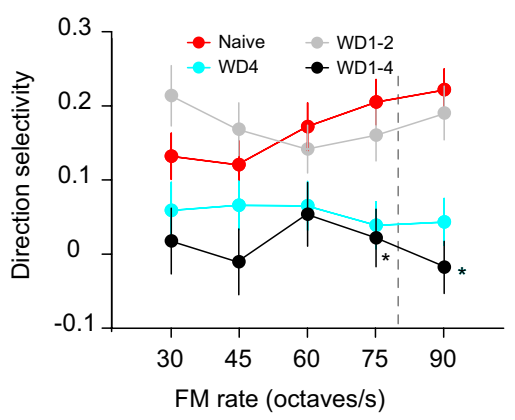

C

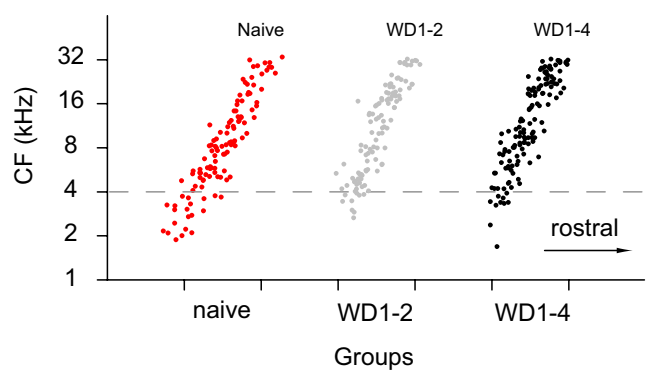

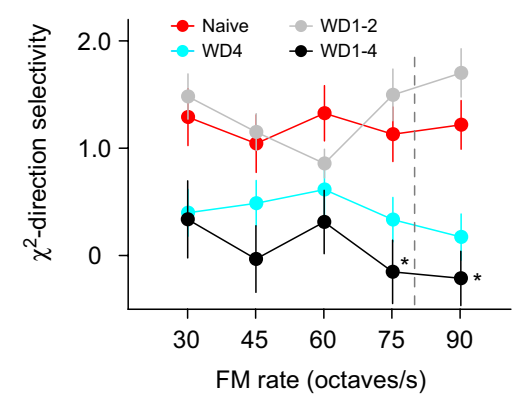

D

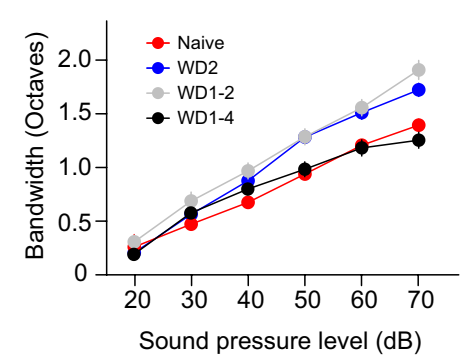

Figure 5. Exposure to downward sweeps in all four windows alters CF distribution and sweep direction selectivity. $\boldsymbol{A}$, The SDSI was down-shifted in animals that had been exposed to downward sweeps in windows 1 through 4 , but not in animals exposed to the sweep in windows 1 and 2. $\boldsymbol{B}, \chi^{2}$ statistic of sweep direction selectivity. $\boldsymbol{C}$, Characteristic frequency distribution along the tonotopic axis. Both window 1-2 and window 1-4 groups had fewer neurons tuned to frequencies $<4 \mathrm{kHz}$. D, Tuning bandwidth as a function of sound pressure levels. The window 1-2 group, but not the window 1- 4 group, showed broadened frequency tuning at $50-70 \mathrm{~dB}$ SPL. ${ }^{*} p<0.05$.

other groups), indicating increased selectivity for the experienced downward FM sweep. Examination of the SDSI of individual neurons revealed that in naive animals, most direction selective neurons preferred upsweeps (Fig. 1C). In the animals that were

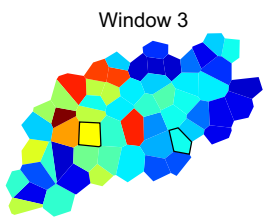

exposed to the downward FM sweep in window 4, fewer neurons selected for upsweeps and more neurons preferred downsweeps (ANOVA group comparison, $p<$ 0.05). Exposure to downsweeps can alter the number of low-CF neurons (see results below and Fig. 3). To avoid potential bias caused by this difference in CFs, we calculated SDSI only for neurons with CFs $>4$ $\mathrm{kHz}$. The results of this subset analysis did not differ from those of analysis of all neurons (ANOVA, $p>0.1$ ). Adult animals $(>3$ months old) that had been exposed to the same downward FM sweep for 4 weeks showed no changes in sweep direction selectivity (Fig. 2) ( $p>0.5$ ).

We also identified the sweep rate that activated the strongest response in each neuron, which we defined as its best FM rate. Initial examination of the best FM rate showed no significant difference among the naive, window 1 , and window 2 groups, or between the window 3 and window 4 groups; we therefore collapsed those conditions for all further analyses. Neurons that had a best FM rate at -90 octaves/s were more frequently observed in window 3/window 4 animals than in naive/window 1/window 2 animals (Fig. $2 C$ ) $\left(\chi^{2}\right.$ test, $\left.p<0.05\right)$. In window 3 and window 4 animals, more neurons had better FM rates at -90 octaves/s than at -30 octaves/s (Fig. 2C) ( $\chi^{2}$ test, $\left.p<0.05\right)$. The downward/upward response ratios were also significantly increased in the window 3/window 4 animals for 75 and 90 octaves/s rates when compared with the slower rates, and when compared with the ratios in the naive/window 1/window 2 animals for the same 75 and 90 octaves/s rates (Fig. 2D) ( $\chi^{2}$ test, $\left.p<0.05\right)$. These results indicate that some cortical neurons shifted their FM rate selectivity to that of the experienced sweep. The sensitive period for FM direction selectivity observed in this study coincides with the maturation of inhibitory areas in the frequency-intensity receptive field (Chang et al., 2005), which may contribute to FM direction selectivity (Zhang et al., 2003; Razak and Fuzessery, 2007).

Exposure to FM sweeps alters the CF and BW of cortical neurons

An analysis of spectral tuning revealed that exposure to the FM sound in window 1 significantly reduced the number of neurons tuned to frequencies $<4 \mathrm{kHz}$ ( $\chi^{2}$ test, $p<0.05)$ (Fig. 3). More neurons in window 1 animals were tuned to $4-8 \mathrm{kHz}$ ( $\chi^{2}$ test, $p<0.05$ ), suggesting that there was a shift from low to high CFs. This effect was not seen in animals exposed to the sound in the later time windows.

The BWs of the animals that were exposed to the FM sweeps in 
window 2 were significantly broader that those of the other groups at high sound pressure levels from 50 to $70 \mathrm{~dB}$ (Fig. 4) (ANOVA, $p<0.05$ ). The effect was seen in neurons of all CF ranges. The BW of auditory cortical neurons undergoes rapid developmental changes in a brief period from P16 to P18 (see results below and Fig. 6). Our results indicate that exposure to broadband stimuli during this period shapes broadly selective receptive fields. Exposure to the same downward FM sweep for 4 weeks did not change cortical CF maps (Fig. 3) or bandwidths (Fig. 4) in adult animals (>3 months).

We examined the auditory cortex of animals that had been exposed to 80 octaves/s downward FM sweeps in windows $1-2$, and found reduced representations of frequencies $<4 \mathrm{kHz}\left(\chi^{2}\right.$ test, $\left.p<0.05\right)$, broadened tuning bandwidth (ANOVA, $p<0.05$ ), but no downward shift of FM direction selectivity (Fig. 5). Animals that had been exposed to the downward FM sweeps in all four windows showed reduced representations of frequencies $<4 \mathrm{kHz}\left(\chi^{2}\right.$ test, $\left.p<0.05\right)$ and an increase in downward FM direction selectivity (Fig. 5) (ANOVA, $p<0.05$ ) in primary audi-

tory cortical neurons. The neuronal tuning bandwidth, however, was not broadened (Fig. 5D) (ANOVA, $p>0.5$ ). Thus, sound experience in window 1 did not prevent subsequent broadening of frequency tuning in window 2 or shift of FM direction selectivity in windows 3 and 4 . Furthermore, although exposure to downward FM sweeps in windows 3 and 4 does not change the BW (Fig. 4) ( $p>0.5$ ), it can renormalize the broadened tuning caused by earlier experience in window 2, suggesting that the plasticity effects induced in different sensitive periods may be interactive (i.e., reduction of BW in windows 3 and 4 depends on the increase of BW in window 2). Results for all six conditions are summarized in Table 1.

\section{Development of cortical responses}

To determine whether the three sensitive periods are correlated with specific developmental events, we examined the development of cortical acoustic representations in naive animals. By the end of window 1, the CF map of the primary auditory cortex of the naive group became finely tonotopic, and AI neurons showed narrow spectral tuning (Fig. 6B), as reported previously (de Villers-Sidani et al., 2007). Most AI neurons represented middle frequencies, with less representation of frequencies $<4 \mathrm{kHz}$ or $>25 \mathrm{kHz}$ (for example, see Fig. 7). Spectral tuning rapidly became broader between P16 and P18 (Fig. 6B), coinciding with the sensitive period for tuning bandwidth. Tuning remained broad throughout windows 3 and 4 . Cortical responses to FM sweeps were direction selective in all four windows, with no significant difference between the strengths of sweep direction selectivity in the four windows (Fig. 6C) $(p>0.5)$. The magnitude of cortical responses to FM sweeps was quantified with the maximum and mean responses to the 10 sweeps used in the experiment (i.e., upward- and downward-modulated sweeps at 30, $45,60,75,90$ octaves/s). Both the maximum and the mean response magnitudes to FM sweeps increased rapidly between P24 to P26, coinciding with the onset of the sensitive period for FM sweep direction selectivity (for example, see Fig. 6C). The coincidences

\begin{tabular}{|c|c|c|c|c|c|c|}
\hline & Window 1 & Window 2 & Window 3 & Window 4 & Window 1-2 & Window 1-4 \\
\hline $\begin{array}{l}\text { CF } \\
B W\end{array}$ & Shifted & Broadened & & & $\begin{array}{l}\text { Shifted } \\
\text { Broadened }\end{array}$ & Shifted \\
\hline SDSI & & & Shifted & Shifted & & Shifted \\
\hline
\end{tabular}

between the emergence/maturation of sound feature representations and the sensitive period of the corresponding features suggest that sensory development and plasticity involve the same cortical substrates.

\section{Discussion}

The results of the present study indicate that primary auditory cortical representations are shaped by different features of the same sound in distinct time windows. We referred to these time windows as sensitive periods in the present study to indicate the different time windows of feature sensitivity within the critical period of heightened cortical plasticity. Thus, the critical period of sensory development in the primary auditory cortex is not a unitary event, but comprises a series of feature-dependent sensitive periods. Sensitive periods for more complex sound features tend to occur later, with each sensitive period approximately coincidental with the emergence or maturation of the cortical representation of the corresponding feature-the sensitive period for the cortical tonotopic CF map reside in window 1, when the cortical map first emerges (de Villers-Sidani et al., 2007); the sensitive period for bandwidth plasticity is located in window 2, when BWs of cortical neurons increase normally; plasticity for FM direction selectivity occurs in windows 3 and 4, when strong cortical responses to FM sounds develops (Zhang et al., 2001; Chang and Merzenich, 2003; Chang et al., 2005; de Villers-Sidani et al., 2007). These findings suggest that the sensitive periods 
A

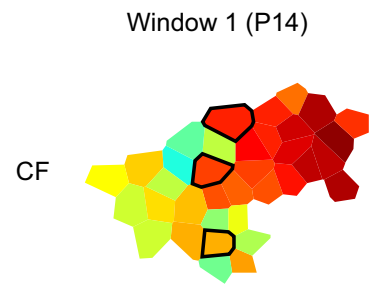

$\mathrm{BW}$
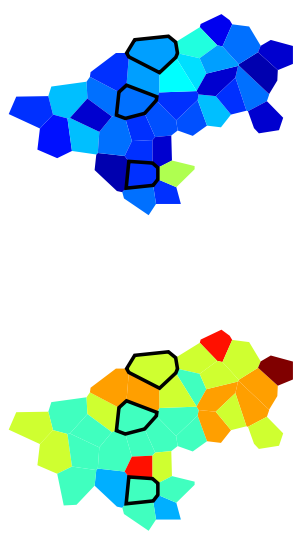

Window $2(\mathrm{P} 18)$
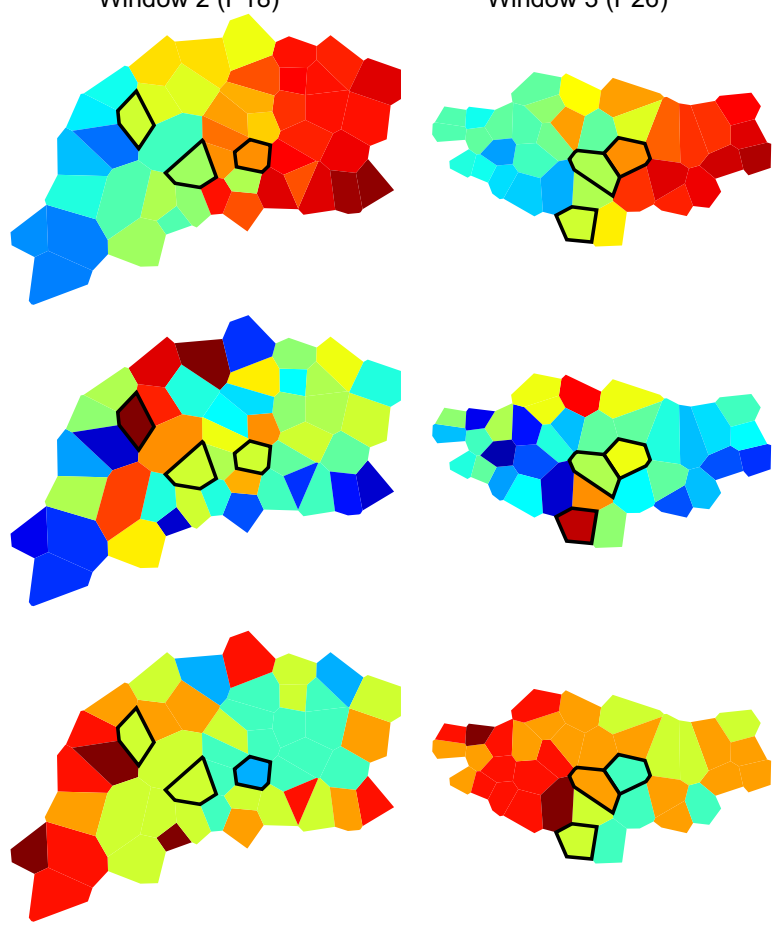

Window 3 (P26)
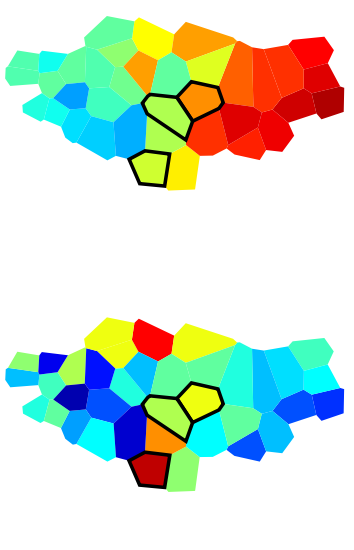
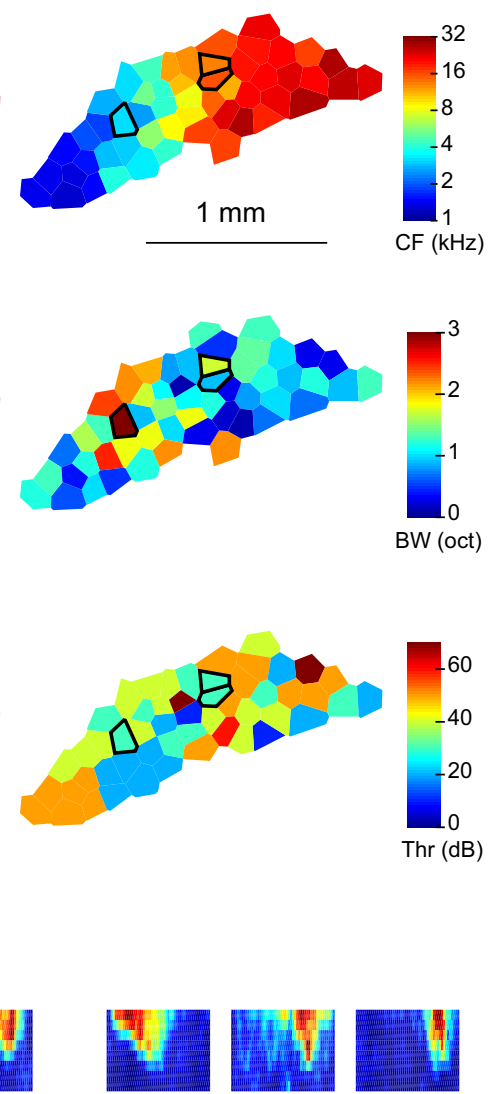

Figure 7. Example cortical maps and receptive fields. A, Representative cortical CF, BW, and response threshold maps obtained in four different developmental windows. B, Representative receptive fields of the neurons whose recording sites are outlined in the maps. The vertical axis of the receptive field plots depicts sound intensity from 0 to $70 \mathrm{~dB} \mathrm{SPL}$. The horizontal axis depicts frequencies from 1 to $32 \mathrm{kHz}$. Neurons in the primary auditory cortex of the P14 animal had narrower frequency tuning than the older animals, while their response thresholds were comparable. The size and shape of the functionally determined primary auditory cortex were more variable in juvenile animals $(<P 40)$ than in adults $(>P 40)$. The difference in Al sizes seen here was not significant $(p>0.05)$.

described in the present study are determined by normal experience-dependent development of sensory representations.

Earlier studies have suggested that the sensitive period for visual direction selectivity may occur later than that for ocular dominance plasticity (Daw and Wyatt, 1976; Li et al., 2006). The two types of plasticity were induced with different procedures in those studies, and involved different sensory inputs, which might have contributed to the observed differences in the timing of the sensitive periods. Experience-dependent plasticity occurs in the auditory brainstem in specific time windows (Clopton and Silverman, 1977; Knudsen, 1985; Yu et al., 2005), and brainstem responses to different types of sounds may develop sequentially (Johnson et al., 2008). Acoustic input/feedback also drives sensory and motor phases of bird song learning in different time windows (Doupe and Kuhl, 1999). Here we have demonstrated that the auditory cortex extracts different information from the same stimuli to shape its cortical circuits in different developmental stages, revealing the progressive nature of the development of complex sound representations. Our findings suggest that sensory inputs must match the processing capacity of the sensory system to improve processing efficiency.

The distinct sensitive periods suggest that CF, BW, and sweep direction selectivity are determined by different cellular circuitries, and cortical plasticity in those response properties occurs in corresponding circuitries (Hensch, 2004). In our experiments, the downward FM sweep activates high-CF neurons before it activates low-CF neurons, possibly enhancing intracortical excitatory connections from high-CF to low-CF neurons through spike-timing dependent plasticity (Yao and Dan, 2001; Fu et al., 2002), and causing an upward shift in CFs. Several recent studies have shown balanced excitatory and inhibitory frequency input to auditory cortical neurons, suggesting that frequency tuning is mediated primarily by excitatory input (Fu et al., 2002; Wehr and Zador, 2003; Zhang et al., 2003; Tan et al., 2004). The slightly lagged inhibitory input may serve to shape sweep selectivity and the precise timing of cortical responses (DeWeese et al., 2003; Zhang et al., 2003; Kandler, 2004; Razak and Fuzessery, 2007). The inhibitory circuit may also be involved in sculpting the excitatory circuit in cortical plasticity (Zheng and Knudsen, 1999; Fagiolini and Hensch, 2000; Fagiolini et al., 2004; Froemke et al., 2007). Recent studies have also suggested that the CF and BW may be determined differently by thalamocortical and intracortical connections (Kaur et al., 2004; Metherate et al., 2005; Liu et al., 2007). Thus, the three sensitive periods in the primary auditory cortex might be mediated separately by thalamocortical, intracortical excitatory, and intracortical inhibitory circuits.

Studies of cognitive development have revealed multiple, hierarchical sensitive periods (Ruben, 1997; Werker and Tees, 2005). The sensitive period for phonological development, for instance, precedes those for syntactic and lexical development. Even 
within phonological development, speech experience alters vowel perception before shaping the perception of acoustically distinct consonants (Kuhl et al., 1992; Polka and Werker, 1994; Werker and Tees, 2005). We may consider static vowel sounds to be analogous to pure tones, and dynamic consonants to be more similar to FM sweeps. The multiple sensitive periods shown in the present study may be relevant to sequential phonological development.

\section{References}

Arimatsu Y, Ishida M (2002) Distinct neuronal populations specified to form corticocortical and corticothalamic projections from layer VI of developing cerebral cortex. Neuroscience 114:1033-1045.

Atencio CA, Blake DT, Strata F, Cheung SW, Merzenich MM, Schreiner CE (2007) Frequency-modulation encoding in the primary auditory cortex of the awake owl monkey. J Neurophysiol 98:2182-2195.

Chang EF, Merzenich MM (2003) Environmental noise retards auditory cortical development. Science 300:498-502.

Chang EF, Bao S, Imaizumi K, Schreiner CE, Merzenich MM (2005) Development of spectral and temporal response selectivity in the auditory cortex. Proc Natl Acad Sci U S A 102:16460-16465.

Clopton BM, Silverman MS (1977) Plasticity of binaural interaction. II. Critical period and changes in midline response. J Neurophysiol 40:1275-1280.

Daw NW, Wyatt HJ (1976) Kittens reared in a unidirectional environment: evidence for a critical period. J Physiol 257:155-170.

de Villers-Sidani E, Chang EF, Bao S, Merzenich MM (2007) Critical period window for spectral tuning defined in the primary auditory cortex (A1) in the rat. J Neurosci 27:180-189.

DeWeese MR, Wehr M, Zador AM (2003) Binary spiking in auditory cortex. J Neurosci 23:7940-7949.

Doupe AJ, Kuhl PK (1999) Birdsong and human speech: common themes and mechanisms. Annu Rev Neurosci 22:567-631.

Fagiolini M, Hensch TK (2000) Inhibitory threshold for critical-period activation in primary visual cortex. Nature 404:183-186.

Fagiolini M, Fritschy JM, Löw K, Möhler H, Rudolph U, Hensch TK (2004) Specific GABAA circuits for visual cortical plasticity. Science 303:1681-1683.

Froemke RC, Merzenich MM, Schreiner CE (2007) A synaptic memory trace for cortical receptive field plasticity. Nature 450:425-429.

Fu YX, Djupsund K, Gao H, Hayden B, Shen K, Dan Y (2002) Temporal specificity in the cortical plasticity of visual space representation. Science 296:1999-2003.

Gaese BH, King I, Felsheim C, Ostwald J, von der Behrens W (2006) Discrimination of direction in fast frequency-modulated tones by rats. J Assoc Res Otolaryngol 7:48-58.

Gao WJ, Newman DE, Wormington AB, Pallas SL (1999) Development of inhibitory circuitry in visual and auditory cortex of postnatal ferrets: immunocytochemical localization of GABAergic neurons. J Comp Neurol 409:261-273

Han YK, Köver H, Insanally MN, Semerdjian JH, Bao S (2007) Early experience impairs perceptual discrimination. Nat Neurosci 10:1191-1197.

Hensch TK (2004) Critical period regulation. Annu Rev Neurosci 27:549-579.

Johnson KL, Nicol T, Zecker SG, Kraus N (2008) Developmental plasticity in the human auditory brainstem. J Neurosci 28:4000-4007.

Kandler K (2004) Activity-dependent organization of inhibitory circuits: lessons from the auditory system. Curr Opin Neurobiol 14:96-104.

Kaur S, Lazar R, Metherate R (2004) Intracortical pathways determine breadth of subthreshold frequency receptive fields in primary auditory cortex. J Neurophysiol 91:2551-2567.

Knudsen EI (1985) Experience alters the spatial tuning of auditory units in the optic tectum during a sensitive period in the barn owl. J Neurosci 5:3094-3109.

Kuhl PK, Williams KA, Lacerda F, Stevens KN, Lindblom B (1992) Linguistic experience alters phonetic perception in infants by 6 months of age. Science 255:606-608.

Kuhl PK, Stevens E, Hayashi A, Deguchi T, Kiritani S, Iverson P (2006) Infants show a facilitation effect for native language phonetic perception between 6 and 12 months. Dev Sci 9:F13-F21.

Larsen DD, Callaway EM (2006) Development of layer-specific axonal ar- borizations in mouse primary somatosensory cortex. J Comp Neurol 494:398-414.

Li Y, Fitzpatrick D, White LE (2006) The development of direction selectivity in ferret visual cortex requires early visual experience. Nat Neurosci 9:676-681.

Linden JF, Liu RC, Sahani M, Schreiner CE, Merzenich MM (2003) Spectrotemporal structure of receptive fields in areas AI and AAF of mouse auditory cortex. J Neurophysiol 90:2660-2675.

Liu BH, Wu GK, Arbuckle R, Tao HW, Zhang LI (2007) Defining cortical frequency tuning with recurrent excitatory circuitry. Nat Neurosci 10:1594-1600.

Loftus WC, Sutter ML (2001) Spectrotemporal organization of excitatory and inhibitory receptive fields of cat posterior auditory field neurons. J Neurophysiol 86:475-491.

Lu T, Liang L, Wang X (2001) Temporal and rate representations of timevarying signals in the auditory cortex of awake primates. Nat Neurosci 4:1131-1138.

Luczyñska A, Dziewiatkowski J, Jagalska-Majewska H, Kowiañski P, Spodnik J, Moryœ J (2003) Postnatal development of the somatosensory thalamocortical projection in rat and rabbit-a combined retrograde transport and stereological comparative study. Folia Morphol (Warsz) 62:129-132.

Metherate R, Aramakis VB (1999) Intrinsic electrophysiology of neurons in thalamorecipient layers of developing rat auditory cortex. Brain Res Dev Brain Res 115:131-144.

Metherate R, Kaur S, Kawai H, Lazar R, Liang K, Rose HJ (2005) Spectral integration in auditory cortex: mechanisms and modulation. Hear Res 206:146-158

Nelken I, Versnel H (2000) Responses to linear and logarithmic frequencymodulated sweeps in ferret primary auditory cortex. Eur J Neurosci 12:549-562.

Polka L, Werker JF (1994) Developmental changes in perception of nonnative vowel contrasts. J Exp Psychol Hum Percept Perform 20:421-435.

Razak KA, Fuzessery ZM (2007) Development of inhibitory mechanisms underlying selectivity for the rate and direction of frequency-modulated sweeps in the auditory cortex. J Neurosci 27:1769-1781.

Ruben RJ (1997) A time frame of critical/sensitive periods of language development. Acta Otolaryngol 117:202-205.

Singh NC, Theunissen FE (2003) Modulation spectra of natural sounds and ethological theories of auditory processing. J Acoust Soc Am 114:3394-3411.

Tan AY, Zhang LI, Merzenich MM, Schreiner CE (2004) Tone-evoked excitatory and inhibitory synaptic conductances of primary auditory cortex neurons. J Neurophysiol 92:630-643.

Wang X, Kadia SC (2001) Differential representation of species-specific primate vocalizations in the auditory cortices of marmoset and cat. J Neurophysiol 86:2616-2620.

Wehr M, Zador AM (2003) Balanced inhibition underlies tuning and sharpens spike timing in auditory cortex. Nature 426:442-446.

Werker JF, Tees RC (1984) Cross-language speech perception: evidence for perceptual reorganization during the first year of life. Infant Behav Dev 7:49-63.

Werker JF, Tees RC (2005) Speech perception as a window for understanding plasticity and commitment in language systems of the brain. Dev Psychobiol 46:233-251.

Yao H, Dan Y (2001) Stimulus timing-dependent plasticity in cortical processing of orientation. Neuron 32:315-323.

Yu X, Wadghiri YZ, Sanes DH, Turnbull DH (2005) In vivo auditory brain mapping in mice with Mn-enhanced MRI. Nat Neurosci 8:961-968.

Zhang LI, Bao S, Merzenich MM (2001) Persistent and specific influences of early acoustic environments on primary auditory cortex. Nat Neurosci 4:1123-1130.

Zhang LI, Bao S, Merzenich MM (2002) Disruption of primary auditory cortex by synchronous auditory inputs during a critical period. Proc Natl Acad Sci U S A 99:2309-2314.

Zhang LI, Tan AY, Schreiner CE, Merzenich MM (2003) Topography and synaptic shaping of direction selectivity in primary auditory cortex. Nature 424:201-205.

Zheng W, Knudsen EI (1999) Functional selection of adaptive auditory space map by GABAA-mediated inhibition. Science 284:962-965. 